\title{
Neutron monitor altitude-dependent yield function and its application to an analysis of neutron-monitor data
}

\section{Alexander Mihsev, ${ }^{a}$ Sergey Koldobskiy, ${ }^{a, b, *}$ Gennady Kovaltsov, ${ }^{c}$ Agnieszka Gil ${ }^{d}$ and Ilya Usoskin ${ }^{a}$}

${ }^{a}$ University of Oulu, Finland

${ }^{b}$ National Research Nuclear University MEPhI, Moscow, Russia

${ }^{c}$ Ioffe Physical-Technical Institute, St. Petersburg, Russia

${ }^{d}$ Siedlce University, Institute of Mathematics, Poland

E-mail: sergey.koldobskiy@oulu.fi

\begin{abstract}
An updated yield function (YF) of a standard NM64 neutron monitor (NM) is computed for different atmospheric depths from sea level to $500 \mathrm{~g} / \mathrm{cm}^{2}(\sim 5.7 \mathrm{~km}$ altitude) and is presented as a full parametrization. The NM YF was computed using the PLANETOCOSMICS simulation tool based on the GEANT4 package, applying the NRLMSISE-00 atmospheric model. The yield function was validated using the cosmic-ray spectra directly measured in space by the AMS-02 experiment during the period May 2011 through May 2017 and confronted with count rates of all NM64-type NMs being in operation during this period. Using this approach, the stability of all selected NMs was analyzed for the period 2011-2017, most of NMs appear very stable and suitable for studies of long-term solar modulation of cosmic rays. However, some NMs suffer from instabilities like trends, apparent jumps, or strong seasonal waves in the count rates.
\end{abstract}

$37^{\text {th }}$ International Cosmic Ray Conference (ICRC 2021)

July 12th-23rd, 2021

Online - Berlin, Germany

\footnotetext{
${ }^{*}$ Presenter
} 


\section{Introduction}

One of the main devices to study the variations of cosmic rays (CRs) as well as other transients [1] is a neutron monitor (NM), which is suited for the registration of nucleonic component of air-shower-cascades induced by primary cosmic rays. NMs were installed around the globe since the 1950s and are still in operation nowadays. Such a long period of their operation makes them a crucial device for the study of cosmic ray variability, both long-term, influenced by solar activity cycles, and sporadic, connected to solar eruptive events and their influence on the heliosphere. Despite the fact that NM is an integral device and its only output is count rate, an analysis of the data from the network of neutron monitors, located in different geographic sites, allows one to reconstruct fluxes and angular distributions of charged particles in the energy region from $\sim 1 \mathrm{GeV}$ [2].

NM data analysis is evolving, previously the only way of analysis was the use of so-called coupling functions [3], but nowadays the NM community uses the NM yield function (YF) $Y_{i}(E)$, which allows one to calculate the NM count rate knowing the spectrum of cosmic-ray particles $J_{i}(E)$ :

$$
N=\frac{1}{\kappa} \sum_{i} \int_{P_{c}}^{\infty} J_{i}(E) Y_{i}(E) d E,
$$

where the summation is over the number of considered cosmic-ray species, $P_{c}$ is the cutoff rigidity of a given NM. A scale factor $\kappa$ is introduced to account for the non-ideality of a given NM, related to electronics, the material of the building where the NM is located.

The most convenient way to calculate the NM yield function is to simulate the particle interaction with the atmosphere and the generation of particle cascades (as pioneered by Debrunner and Brunberg [4]), as well as particle interaction and registration inside the NM. For the first time using the modern Monte-Carlo particle simulations, this approach was used by Clem and Dorman [5]. In consecutive years, several other models were developed [6-9], which were based on Monte-Carlo simulations and/or latitude surveys. However, it was not possible to directly verify the NM YFs on the basis of satellite measurements due to the lack of the latter. The situation has changed in 2018 when the AMS-02 data on proton and helium fluxes for the period 2011-2017 with Bartels rotation time resolution were published [10]. These data (together with precise measurements of heavier-than-helium cosmic rays) allowed us to verify yield functions using direct measurements in the space for period from May 2011 to May 2017 covering the solar activity maximum around 2014 [11]. Validation made it clear that the NM YF by Mishev et al. [8] shows the best performance since the scale factors obtained with this function are stable in time and there are no trends connected to the phase of the solar cycle. However, this YF was not suited for the count rate calculation of high-altitude NMs. We have performed additional simulations and computations in order to expand the YF coverage to different atmospheric depths and apply it to the analysis of NMs. In this paper we shortly discuss the main steps of this analysis, while details are given elsewhere [12].

\section{NM YF computation}

The NM YF can be generally computed as follows:

$$
Y i(E, h)=G(E) \sum_{i} \iint S_{j}\left(E^{\prime}, \theta\right) \cdot F_{i, j}\left(E, h, E^{\prime}, \theta\right) d E^{\prime} d \Omega
$$


where $G(E)$ is the geometrical correction factor, accounting for the finite lateral expansion of the CR-induced atmospheric cascades and the detector's electronic dead time; $S_{j}\left(E^{\prime}, \theta\right)$ is the detector's response function, equal to the geometrical area times the registration efficiency for a secondary particle of type $j$ with energy $E^{\prime}$ impinging on the detector with the zenith angle $\theta ; F_{i, j}\left(E, h, E^{\prime}, \theta\right)$ is the differential flux of secondary particles of type $j$ (neutrons, protons, muons, and pions) for a primary particle of type $i$ with kinetic energy per nucleon $E$, impinging isotropically on the top of the atmosphere; and the integration is over the energy of secondary particles $E^{\prime}$ and solid angle $\Omega$. All the computations were done for the standard 6NM64 NM [13]. Another type of NM, called IGY [14], was the primary type of detectors before 1964 and is not considered here, since it has different sensitivity to secondary particles. However we expect that the IGY YF is similar to that of 6NM64 [5], up to a scaling factor.

Here, the propagation and interaction of primary particles in the atmosphere were simulated with the PLANETOCOSMICS simulation tool [15] based on the GEANT4 package, considering the NRLMSISE-00 atmospheric model. The simulation scheme was the same as in the previous computations by Mishev et al. [8]. First, we calculated fluxes of secondary particles at prescribed atmospheric depths and then convoluted them with the registration efficiency (Eq. 2). Here we used the NM registration efficiency according to [5], considering secondary neutrons, protons, positive and negative pions, and muons that contribute to the counts of the device. The computations were carried out separately for primary cosmic-ray protons and helium nuclei with an isotropic incidence in a wide energy range from $\sim 0.5 \mathrm{GeV} /$ nucleon to $1 \mathrm{TeV} /$ nucleon.

Calculations were performed for atmospheric depths of $1000,900,700$, and $500 \mathrm{~g} / \mathrm{cm}^{2}$ that (together with original YF by Mishev et al. [8] computed for $1033 \mathrm{~g} / \mathrm{cm}^{2}$ ) correspond to the full range of NM locations from sea-level to high altitudes.

The computed YF was parametrized in an easy-to-use way, allowing to use a simple analytical

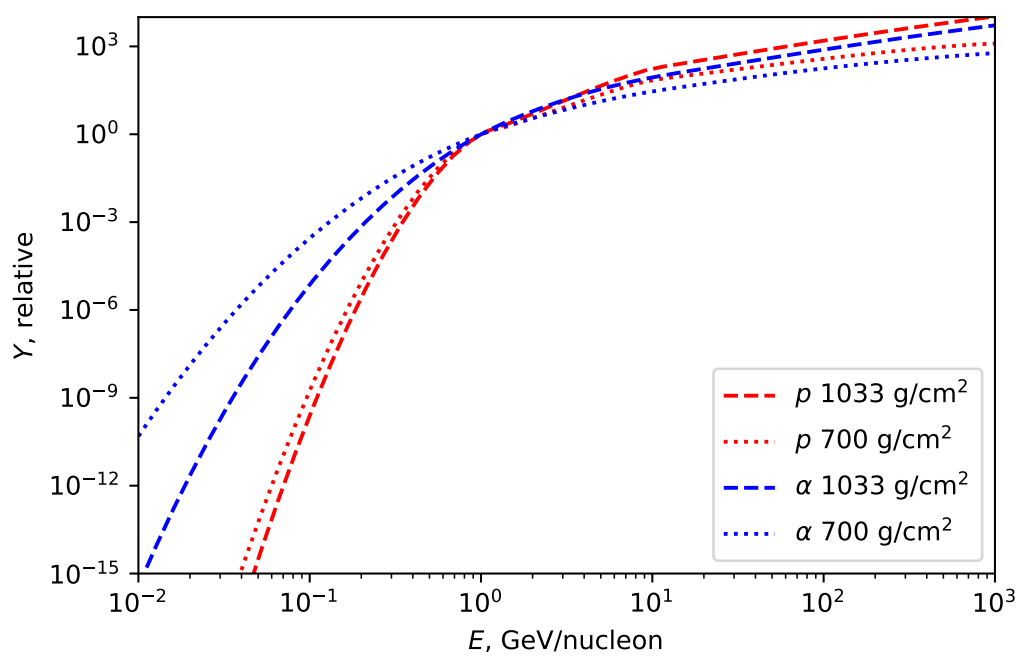

Figure 1: YF for standard 6NM64 NM for primary proton (red lines) and helium (blue), computed for atmospheric depths of 1033 (dashed) and $700 \mathrm{~g} / \mathrm{cm}^{2}$ (dotted). All YFs are scaled to unity at $1 \mathrm{GeV} /$ nucleon. 
expression for $Y_{i}(E, h)$ calculation:

$$
\begin{array}{r}
\ln \left(\frac{Y(h, E)}{Y(1000, E)}\right)=A(R) \cdot(1000-h)^{2}+B(R) \cdot(1000-h), \\
\ln (Y(1000, E))=\sum_{l=0}^{3} a_{l}(\ln (R))^{l}, \\
A(R)(\text { or } B(R))=\sum_{j=0}^{5} b_{j}(\ln (R))^{j},
\end{array}
$$

where $E$ is a particle energy and $R$ is the corresponding rigidity value, $\mathrm{h}$ is the altitude above the sea-level for NM in a given location. Numeric values of coefficients $a_{l}$ and $b_{j}$ are given elsewhere [12]. An example of obtained parametrization is given in Fig. 1, where YFs for protons and helium nuclei for two atmospheric depths are given.

\section{Calibration of NM network using AMS-02 data}

We applied the derived yield function to the data of the global NM network similarly to [11, 16]. Data were collected using Neutron Monitor Data Base (NMDB, https://www . nmdb.eu/), IZMIRAN database (http://cr0.izmiran.ru/common/links.htm) and dedicated servers for several NMs (recently, the dedicated study of the data availability and quality along different databases was performed by Väisänen et al. [17]). NMs active during the period 2011-2017 (corresponding to the period of CR observations by AMS-02 [10]) were considered, moreover, only NMs having the observational data during at least half of the considered 2011-2017 timespan were included in the analysis, this condition was satisfied by $42 \mathrm{NMs}$.

Daily NM count rates were integrated on Bartels rotation time basis to make them comparable with AMS-02 data. Heavier-than-helium nuclei species were represented by helium nuclei, scaled according to the number of nucleons, with the same energy per nucleon. Their combined spectrum was reconstructed using experimental data from AMS-02 and another space-borne experiments and rebinned correspondingly, so that for each helium nuclei energy bin the corresponding contribution of heavier-than-helium nuclei was calculated and added [11]. After that, the fluxes of protons and helium nuclei (together with heavy-than-helium CR fluxes data) were used for the computation of theoretical NM response (Eq. 1) and were compared to NM real data. For each NM, the most stable count rate series among different databases was taken into the analysis.

Several characteristics of NMs were checked. First, the scale factor $\kappa$ was reconstructed as a ratio between theoretical and measured count rates, results of this reconstruction are given in Fig. 2, where a diagram of cutoff rigidity vs. altitude is shown, each circle corresponds to NM, and the size of the circle to the value of the scaling factor $\kappa$. Typically it is in the range from 0.8 to 1.2, that shows that considered YF is valid for an analysis of NMs located at different latitudes (and corresponding cutoff rigidities) and altitudes. Slightly greater values of $\kappa$ are observed for SNM-15 NM tubes produced in the former Soviet Union (denoted with red circles), since these NM tubes possess lower efficiency (by 15-20\%) [18]. However, several NMs (e.g., CALM and THUL) exhibit significantly higher values of $\kappa$. 


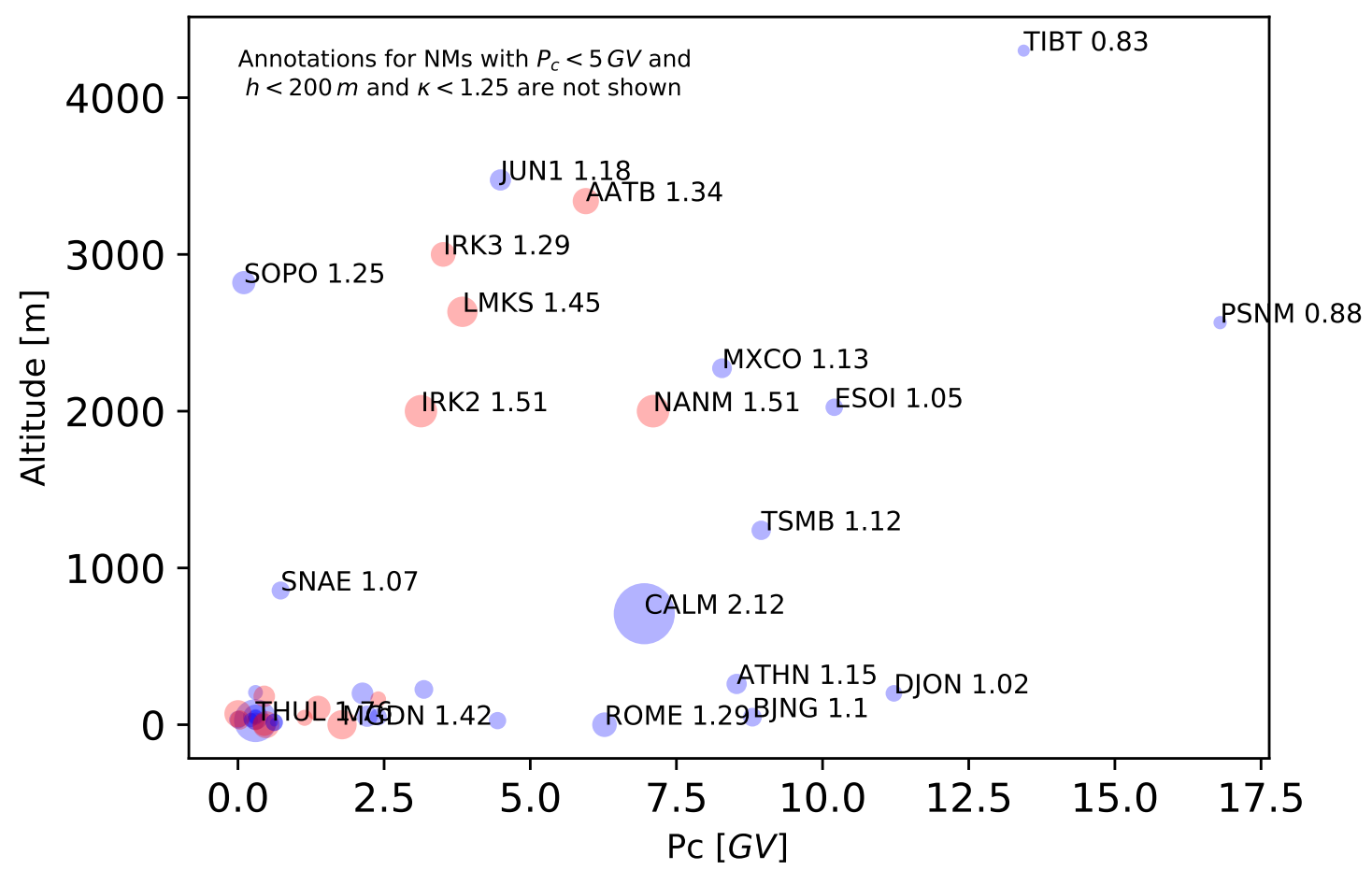

Figure 2: Cutoff rigidity $P_{c}$ and altitude $h$ in meters of NMs considered in this analysis, each point corresponds to one NM. Size of the point corresponds to the value of scaling factor $\kappa$, the color corresponds to the type of NM: blue are standard 6NM64, red are their modifications SNM-15, produced in Soviet Union. Annotations (together with values of $\kappa$ ) are given for selected NMs.

Second, the variation of scale factor over considered timespan was calculated; it is expected that stable NMs should have a small variation of $\kappa$. Most of the analysed NMs reveal small variations of $\kappa$ (less than 1\%) with several exceptions with unstable data. A considerably long (79 Bartels rotations) period of observations allowed us to study the nature of scale factor $K$ variations, such as possible permanent increases (or decreases) of NM count rate, sudden jumps, or annual variations (probably due to the snow coverage). We find that about half of analysed NMs do not have any notable features in $\kappa$ variation over time during the studied timespan, while the second half of NMs show trends (for example, BRBG), apparent jumps (DRBS, IRK2, NANM), and seasonal waves (ESOI, JUN1). The level of these instabilities is also different and is typically not high. Detailed study of scale factor stability for each NM for period 2011-2017 can be found in [12].

We emphasize that we employed NM records retrieved form the corresponding databases, therefore, sudden jumps in the scale factor can be due to either malfunctions of NMs and/or errors in the databases. On the other hand, trends and seasonal waves are hardly explainable with the data errors, thus are most likely clearly observable experimental effects.

\section{Conclusion}

In this work, we presented a new neutron monitor yield function, calculated for different atmospheric depths from 500 to $1033 \mathrm{~g} / \mathrm{cm}^{2}$ [12]. The calculation was performed for protons and 
helium nuclei (heavier-than-helium elements can be effectively described as additional scaling for helium nuclei). The obtained yield function is parametrized in an easy-to-use way.

Also, the validation of experimental NM count rates using the obtained NM YF and AMS-02 experimental data is performed for the period from 2011 to 2017. Several NM databases are used in the analysis, about half of NMs shows very good performance during the studied period of time, while the second half suffer from seasonal waves, jumps, and trends in data.

Obtained results form a solid basis for further NM analysis with the aim of a better understanding of the variability of cosmic rays, both periodic and sporadic and give robust basis to improve and verify the developed methods for precise study of the spectra and angular distribution of solar protons [19].

\section{Acknowledgments}

PIs and teams of the experiments whose data were used here are gratefully acknowledged. This work was partially supported by the Academy of Finland (Projects HEAIM 314982 and 316223; QUASARE 330064 and ESPERA 321882), the Russian Science Foundation project no. 20-7210170, as well as by the International Space Science Institute support to International Team 441: High EneRgy sOlar partICle Events Analysis (HEROIC).

\section{References}

[1] A. Gil, G.A. Kovaltsov, V.V. Mikhailov, A. Mishev, S. Poluianov and I.G. Usoskin, An Anisotropic Cosmic-Ray Enhancement Event on 07-June-2015: A Possible Origin, Solar Physics 293 (2018) 154.

[2] A.L. Mishev, S.A. Koldobskiy, L.G. Kocharov and I.G. Usoskin, GLE \# 67 Event on 2 November 2003: An Analysis of the Spectral and Anisotropy Characteristics Using Verified Yield Function and Detrended Neutron Monitor Data, Solar Physics 296 (2021) 79.

[3] L.I. Dorman and V.G. Yanke, The coupling functions of NM-64 neutron supermonitor, Proceedings of the 17th ICRC, Paris 4 (1981) 326.

[4] H. Debrunner and E. A. Brunberg, Monte Carlo calculation of the nucleonic cascade in the atmosphere, Canadian Journal of Physics 46 (1968) S1069.

[5] J.M. Clem and L.I. Dorman, Neutron monitor response functions, Space Science Reviews 93 (2000) 335.

[6] E.O. Flückiger, M.R. Moser, B. Pikard, R. Butikofer and L. Desorgher, A parameterized neutron monitor yield function for space weather applications, in Proceedings of the 30th International Cosmic Ray Conference, G.M.-T. Rogelio Caballero, Juan Carlos D’Olivo and J.F.V.-G. Lukas Nellen, Federico A. Sánchez, eds., (Mexico City), pp. 289-282, 2008, http://indico.nucleares.unam.mx/event/4/session/78/contribution/1182/material/paper/0.pdf.

[7] R.A. Caballero-Lopez and H. Moraal, Cosmic-ray yield and response functions in the atmosphere, Journal of Geophysical Research: Space Physics 117 (2012) A12103. 
[8] A.L. Mishev, I.G. Usoskin and G.A. Kovaltsov, Neutron monitor yield function: New improved computations, Journal of Geophysical Research: Space Physics 118 (2013) 2783.

[9] P.-S. Mangeard, D. Ruffolo, A. Sáiz, S. Madlee and T. Nutaro, Monte Carlo simulation of the neutron monitor yield function, Journal of Geophysical Research: Space Physics 121 (2016) 7435 .

[10] M. Aguilar, L. Ali Cavasonza, B. Alpat, G. Ambrosi, L. Arruda, N. Attig et al., Observation of Fine Time Structures in the Cosmic Proton and Helium Fluxes with the Alpha Magnetic Spectrometer on the International Space Station, Physical Review Letters 121 (2018) 051101.

[11] S.A. Koldobskiy, V. Bindi, C. Corti, G.A. Kovaltsov and I.G. Usoskin, Validation of the neutron monitor yield function using data from AMS-02 experiment, 2011-2017, Journal of Geophysical Research: Space Physics 124 (2019) 2367 [1904 . 01929].

[12] A.L. Mishev, S.A. Koldobskiy, G.A. Kovaltsov, A. Gil and I.G. Usoskin, Updated Neutron-Monitor Yield Function: Bridging Between In Situ and Ground-Based Cosmic Ray Measurements, Journal of Geophysical Research: Space Physics 125 (2020) e2019JA027433.

[13] C.J. Hatton and H. Carimichael, Experimental investigation of the NM-64 neutron monitor, Canadian Journal of Physics 42 (1964) 2443.

[14] J.A. Simpson, The Cosmic Ray Nucleonic Component: The Invention and Scientific Uses of the Neutron Monitor, in Space Science Reviews, vol. 93, pp. 11-32 (2000), DOI.

[15] L. Desorgher, E.O. Flückiger, M. Gurtner, M.R. Moser and R. Bütikofer, ATMOCOSMICS: A geant 4 code for computing the interaction of cosmic rays with the Earth's atmosphere, International Journal of Modern Physics A 20 (2005) 6802.

[16] S.A. Koldobskiy, G.A. Kovaltsov and I.G. Usoskin, A Solar Cycle of Cosmic Ray Fluxes for 2006-2014: Comparison between PAMELA and Neutron Monitors, Journal of Geophysical Research: Space Physics 123 (2018) 4479.

[17] P. Väisänen, I. Usoskin and K. Mursula, Seven Decades of Neutron Monitors (1951-2019): Overview and Evaluation of Data Sources, Journal of Geophysical Research: Space Physics 126 (2021) e2020JA028941.

[18] A. Gil, I.G. Usoskin, G.A. Kovaltsov, A.L. Mishev, C. Corti and V. Bindi, Can we properly model the neutron monitor count rate?, Journal of Geophysical Research: Space Physics 120 (2015) 7172.

[19] A.L. Mishev, S.A. Koldobskiy, I.G. Usoskin, L.G. Kocharov and G.A. Kovaltsov, Application of the Verified Neutron Monitor Yield Function for an Extended Analysis of the GLE \# 71 on 17 May 2012, Space Weather 19 (2021) e2020SW002626. 